\title{
INVESTIGATION OF G-NETWORK WITH SIGNALS AT TRANSIENT BEHAVIOR
}

\author{
Mikhail Matalytski ${ }^{1}$, Victor Naumenko ${ }^{2}$ \\ ${ }^{I}$ Institute of Mathematics, Czestochowa University of Technology \\ Częstochowa, Poland \\ ${ }^{2}$ Faculty of Mathematics and Computer Science, Grodno State University \\ Grodno, Belarus \\ Im.matalytski@gmail.com, ${ }^{2}$ victornn86@gmail.com
}

\begin{abstract}
G-queueing network with positive messages and signals at transient behavior is considered. A system of difference-differential equations for the state probabilities of the network is obtained. To find them and the average characteristics of the network a technique was applied based on the use of apparatus of multivariate generating functions. An expression for the generating function was obtained. An example is calculated.
\end{abstract}

Keywords: G-queueing network, transient behavior, transient behavior, state probabilities, generating function

\section{General information}

Previously were investigated queueing networks (QN) with negative customers (G-network). For such networks an analysis was performed at transient behavior $[1,2]$. A system of difference-differential equations (DDE) for the state probabilities was obtained and a solution was found.

The impact of the environment on the queue process of positive messages can benefit not only the negative messages that are simply destroying one or more positive messages in the queueing system QN (QS), but also coming from outside signals-triggers, the effect of which is to move the instant the positive message of this system in some other network system. Thus, the trigger, unlike the negative message, does not destroy the positive message but merely immediately moves it with a specified probability of the system in some other network system. G-network with triggers at the stationary behaviour has been studied in [3, 4]. Note that in some studies [5] the concept of a trigger and signal are identified. Clearly, this is a mixture of terms, however, this fact must be taken into account when studying the works on G-networks.

QS with signals (negative messages and/or triggers) are used in the analytical modeling of information technology systems and networks, with negative messages possibly arising, for example, for simulation of computer viruses, and the introduction of triggers to manage the load on the network. G-network is also widely used to model neural networks, and drive signals are modeled by positive messages, 
and braking signals - by negative messages. Further details on the practical use of G-networks with signals are described in [6].

So, consider an open queueing G-network with $n$ single-line QS. In QS $S_{i}$ from the outside (from the system $S_{0}$ ) an incoming flow of positive (normal) of messages intensity of $\lambda_{0 i}^{+}$and Poisson flow of signals intensity of $\lambda_{0 i}^{-}, i=\overline{1, n}$. All flows of messages entering the network are independent. The service time of the positive messages in the QS $S_{i}$ exponentially distributed with mean $\mu_{i}, i=\overline{1, n}$. After servicing the positive message in QS $S_{i}$, it is sent to the QS $S_{j}$ with probability $p_{i j}^{+}$again as positive message, with probability $p_{i j}^{-}$- as a signal and with probability $p_{i 0}=1-\sum_{j=1}^{n}\left(p_{i j}^{+}+p_{i j}^{-}\right)$is leaving from the network to the external environment, $i, j=\overline{1, n}$.

Thus, in the network are circulating not only positive messages, and signals too. Signal, coming in an empty system $S_{i}$ (in which there are no positive messages), does not have any impact on the network and immediately disappears from it. Otherwise, if the system $S_{i}$ is not empty, when it receives a signal, there can occur following events: incoming signal instantly moves the positive message from the system $S_{i}$ into the system $S_{j}$ with probability $q_{i j}$, in this case, signal is referred to as a trigger; or with probability $q_{i 0}=1-\sum_{j=1}^{n} q_{i j}$ signal is triggered by a negative message and destroys in QS $S_{i}$ positive message [3]. The state of the network meaning the vector $k(t)=(k, t)=\left(k_{1}, k_{2}, \ldots, k_{n}, t\right)$, where $k_{i}$ - the number of messages at the moment of time $t$ at the system $S_{i}, i=\overline{1, n}$.

It is necessary to find network state probabilities and average characteristics of the network at transient behavior.

\section{Network state probabilities}

Lemma. State probabilities of considered network satisfy the system of DDE:

$$
\begin{gathered}
\frac{d P(k, t)}{d t}=-\sum_{i=1}^{n}\left[\lambda_{0 i}^{+}+\left(\lambda_{0 i}^{-}+\mu_{i}\right) u\left(k_{i}\right)\right] P(k, t)+\sum_{i=1}^{n}\left\{\lambda_{0 i}^{+} u\left(k_{i}\right) P\left(k-I_{i}, t\right)+\right. \\
+\left(\mu_{i} p_{i 0}+\lambda_{0 i}^{-} q_{i 0}\right) P\left(k+I_{i}, t\right)+\sum_{j=1}^{n}\left[\mu_{i}\left(1-u\left(k_{j}\right)\right) p_{i j}^{-} P\left(k+I_{i}, t\right)+\right. \\
+\left(\mu_{i} p_{i j}^{+}+\lambda_{0 i}^{-} q_{i j}\right) u\left(k_{j}\right) P\left(k+I_{i}-I_{j}, t\right)+\mu_{i} p_{i j}^{-} q_{j 0} P\left(k+I_{i}+I_{j}, t\right)+ \\
\left.\left.+\sum_{s=1}^{n} \mu_{i} p_{i j}^{-} q_{j s} u\left(k_{s}\right) P\left(k+I_{i}+I_{j}-I_{s}, t\right)\right]\right\}
\end{gathered}
$$


where $I_{i}$ - a vector of dimension $n$, consisting of zeros, except for the component with number of $i$, which is equal to $1, i=\overline{1, n} ; u(x)=\left\{\begin{array}{l}1, x>0 \\ 0, x \leq 0\end{array}\right.$ - Heaviside function.

Proof. In view of the exponential service times of messages, a random process $k(t)=(k, t)$ is a Markov chain with a countable number of states. The possible transitions in the state $(k, t+\Delta t)$ for the time $\Delta t$ :

$\checkmark$ from the state $\left(k-I_{i}, t\right)$, in this case into QS $S_{i}$ during time $\Delta t$ coming positive message with probability $\lambda_{0 i}^{+} u\left(k_{i}\right) \Delta t+o(\Delta t)$;

$\checkmark$ from the state $\left(k+I_{i}, t\right)$, while a positive message goes out from the network to the external environment or goes to QS $S_{j}$ as signal, if it was not messages, or arriving in the QS $S_{i}$ signal triggered as a negative message and destroys the QS $S_{i}$ positive message; the probability of such event is equal to $\left(\mu_{i} p_{i 0}+\lambda_{0 i}^{-} q_{i 0}+\mu_{i}\left(1-u\left(k_{j}\right)\right) p_{i j}^{-}\right) \Delta t+o(\Delta t)$;

$\checkmark$ from the state $\left(k+I_{i}-I_{j}, t\right)$, in this case, after the servicing of positive message in the QS $S_{i}$ it goes to the QS $S_{j}$ again as a positive message or arriving in the QS $S_{i}$ signal immediately moves the positive message from the system $S_{i}$ to the system $S_{j}$; the probability of such event is equal to $\left(\mu_{i} p_{i j}^{+}+\lambda_{0 i}^{-} q_{i j}\right) u\left(k_{j}\right) \Delta t+o(\Delta t)$;

$\checkmark$ from the state $\left(k+I_{i}+I_{j}, t\right)$, while after the servicing of positive message in the QS $S_{i}$ it goes to the QS $S_{j}$ as signal, which is triggered as a negative message, destroyed in $S_{j}$ positive message; the probability of such event is equal to $\mu_{i} p_{i j}^{-} q_{j 0} \Delta t+o(\Delta t)$

$\checkmark$ from the state $\left(k+I_{i}+I_{j}-I_{s}, t\right)$, in this case, after the completion of the service message in the QS $S_{i}$, it goes to the QS $S_{j}$ as signal, which immediately moves the positive message from the system $S_{j}$ to the system $S_{s}$; the probability of such event is equal to $\mu_{i} p_{i j}^{-} q_{j s} u\left(k_{s}\right) \Delta t+o(\Delta t)$;

$\checkmark$ from the state $(k, t)$, while in each QS $S_{i}, i=\overline{1, n}$, no positive messages arrive, any signals and which during $\Delta t$ do not to service any message; the probability of such event is equal to $\left(1-\sum_{i=1}^{n}\left[\lambda_{0 i}^{+}+\left(\lambda_{0 i}^{-}+\mu_{i}\right) u\left(k_{i}\right)\right]\right) \Delta t+o(\Delta t)$; of the remaining states with a probability $o(\Delta t)$. 
Then, using the formula of total probability and taking the limit $\Delta t \rightarrow 0$, we obtain a system of equations for the state probabilities of the network (1). The lemma is proved.

Suppose that all systems of the network are operating in high load, i.e. $k_{i}(t)>0$, $\forall t>0, i=\overline{1, n}$, then the system of DDE (1) takes the form

$$
\begin{gathered}
\frac{d P(k, t)}{d t}=-\sum_{i=1}^{n}\left[\lambda_{0 i}^{+}+\lambda_{0 i}^{-}+\mu_{i}\right] P(k, t)+ \\
+\sum_{i=1}^{n}\left\{\lambda_{0 i}^{+} P\left(k-I_{i}, t\right)+\left(\mu_{i} p_{i 0}+\lambda_{0 i}^{-} q_{i 0}\right) P\left(k+I_{i}, t\right)+\right. \\
+\sum_{j=1}^{n}\left[\left(\mu_{i} p_{i j}^{+}+\lambda_{0 i}^{-} q_{i j}\right) P\left(k+I_{i}-I_{j}, t\right)+\mu_{i} p_{i j}^{-} q_{j 0} P\left(k+I_{i}+I_{j}, t\right)+\right. \\
\left.\left.+\sum_{s=1}^{n} \mu_{i} p_{i j}^{-} q_{j s} P\left(k+I_{i}+I_{j}-I_{s}, t\right)\right]\right\} .
\end{gathered}
$$

Denote by $\Psi_{n}(z, t)$, where $z=\left(z_{1}, z_{2}, \ldots, z_{n}\right), n$-dimensional generating function:

$$
\Psi_{n}(z, t)=\sum_{k_{1}=0 k_{2}=0}^{\infty} \sum_{k_{n}=0}^{\infty} \ldots \sum^{\infty} P\left(k_{1}, k_{2}, . ., k_{n}, t\right) z_{1}^{k_{1}} z_{2}^{k_{2}} \cdot \ldots \cdot z_{n}^{k_{n}}=\sum_{k_{1}=0 k_{2}=0}^{\infty} \sum_{k_{n}=0}^{\infty} \ldots \sum_{k_{n}}^{\infty} P(k, t) \prod_{i=1}^{n} z_{i}^{k_{i}} .
$$

Multiplied (2) on $\prod_{l=1}^{n} z_{l}^{k_{l}}$ and adding together all possible values $k_{l}$ from 1 to $+\infty, l=\overline{1, n}$, obtain the inhomogeneous linear differential equations

$$
\begin{gathered}
\frac{d \Psi_{n}(z, t)}{d t}=-\left[\sum_{i=1}^{n}\left(\lambda_{0 i}^{+}+\lambda_{0 i}^{-}+\mu_{i}\right)-\sum_{i=1}^{n} \lambda_{0 i}^{+} z_{i}-\sum_{i=1}^{n} \frac{\mu_{i} p_{i 0}+\lambda_{0 i}^{-} q_{i 0}}{z_{i}}-\right. \\
\left.-\sum_{i, j=1}^{n}\left(\mu_{i} p_{i j}^{+}+\lambda_{0 i}^{-} q_{i j}\right) \frac{z_{j}}{z_{i}}-\sum_{i, j=1}^{n} \mu_{i} p_{i j}^{-} q_{j 0} \frac{1}{z_{i} z_{j}}-\sum_{i, j=1}^{n} \mu_{i} \sum_{s=1}^{n} p_{i j}^{-} q_{j s} \frac{z_{s}}{z_{i} z_{j}}\right] \Psi_{n}(z, t)- \\
-\sum_{i=1}^{n} \frac{\mu_{i} p_{i 0}+\lambda_{0 i}^{-} q_{i 0}}{z_{i}} \sum_{\substack{k_{j}=0 \\
j=1, n, j \neq i}}^{\infty} P\left(k_{1}, \ldots, k_{i-1}, 0, k_{i+1} . ., k_{n}, t\right) \prod_{\substack{l=1 \\
l \neq i}}^{n} z_{l}^{k_{l}}- \\
-\sum_{i, j=1}^{n}\left(\mu_{i} p_{i j}^{+}+\lambda_{0 i}^{-} q_{i j}\right) \frac{z_{j}}{z_{i}} \sum_{\substack{k_{m}=0 \\
m=1, n, m \neq j}}^{\infty} P\left(k_{1}, \ldots, k_{i-1}, 0, k_{i+1}, \ldots, k_{n}, t\right) \prod_{\substack{l=1 \\
l \neq i}}^{n} z_{l}^{k_{l}}- \\
-\sum_{i, j=1}^{n} \frac{\mu_{i} p_{i j}^{-} q_{j 0}}{z_{i} z_{j}} \sum_{\substack{k_{m}=0 \\
m=1, n, m \neq j}}^{\infty} P\left(k_{1}, \ldots, k_{i-1}, 0, k_{i+1}, \ldots, k_{n}, t\right) \prod_{\substack{l=1 \\
l \neq i}}^{n} z_{l}^{k_{l}}-
\end{gathered}
$$




$$
-\sum_{i, j=1}^{n} \mu_{i} \sum_{s=1}^{n} p_{i j}^{-} q_{j s} \frac{z_{s}}{z_{i} z_{j}} \sum_{\substack{k_{m}=0 \\ m=1, n, m \neq j}}^{\infty} P\left(k_{1}, \ldots, k_{i-1}, 0, k_{i+1}, \ldots, k_{n}, t\right) \prod_{\substack{l=1 \\ l \neq i}}^{n} z_{l}^{k_{l}} .
$$

Since all of the QS network operate under high load conditions, the last four expressions in the form of the sums in equation (3) will be zero, and it becomes homogeneous:

$$
\begin{aligned}
& \frac{d \Psi_{n}(z, t)}{d t}=-\left[\sum_{i=1}^{n}\left(\lambda_{0 i}^{+}+\lambda_{0 i}^{-}+\mu_{i}\right)-\sum_{i=1}^{n} \lambda_{0 i}^{+} z_{i}-\sum_{i=1}^{n} \frac{\mu_{i} p_{i 0}+\lambda_{0 i}^{-} q_{i 0}}{z_{i}}-\right. \\
& \left.-\sum_{i, j=1}^{n}\left(\mu_{i} p_{i j}^{+}+\lambda_{0 i}^{-} q_{i j}\right) \frac{z_{j}}{z_{i}}-\sum_{i, j=1}^{n} \mu_{i} p_{i j}^{-} q_{j 0} \frac{1}{z_{i} z_{j}}-\sum_{i, j=1}^{n} \mu_{i} \sum_{s=1}^{n} p_{i j}^{-} q_{j s} \frac{z_{s}}{z_{i} z_{j}}\right] \Psi_{n}(z, t) .
\end{aligned}
$$

Its solution has the form

$$
\begin{gathered}
\Psi_{n}(z, t)=C_{n} \exp \left\{-\left[\sum_{i=1}^{n}\left(\lambda_{0 i}^{+}+\lambda_{0 i}^{-}+\mu_{i}\right)-\sum_{i=1}^{n} \lambda_{0 i}^{+} z_{i}-\sum_{i=1}^{n} \frac{1}{z_{i}}\left(\mu_{i} p_{i 0}+\lambda_{0 i}^{-} q_{i 0}-\right.\right.\right. \\
\left.\left.\left.-\left(\sum_{j=1}^{n}\left(\mu_{i} p_{i j}^{+}+\lambda_{0 i}^{-} q_{i j}\right) z_{j}+\mu_{i} \sum_{j=1}^{n} \frac{1}{z_{j}}\left(p_{i j}^{-} q_{j 0}+\sum_{s=1}^{n} p_{i j}^{-} q_{j s} z_{s}\right)\right)\right)\right] t\right\} .
\end{gathered}
$$

We assume that at the initial moment of time network is in state $\left(\alpha_{1}, \alpha_{2}, \ldots, \alpha_{n}, 0\right), \alpha_{i}>0, i=\overline{1, n}$,

$$
P\left(\alpha_{1}, \alpha_{2}, \ldots, \alpha_{n}, 0\right)=1, P\left(k_{1}, k_{2}, \ldots, k_{n}, 0\right)=0, \forall \alpha_{i} \neq k_{i}, i=\overline{1, n} .
$$

Then the initial condition for the last equation (4) will be $\Psi_{n}(z, 0)=P\left(\alpha_{1}, \alpha_{2}, \ldots, \alpha_{n}, 0\right) \prod_{l=1}^{n} z_{l}^{\alpha_{l}}=\prod_{l=1}^{n} z_{l}^{\alpha_{l}}$. Using it, we obtain $C_{n}=1$.

Thus, the expression for the generating function $\Psi_{n}(z, t)$ has the form

$$
\begin{aligned}
& \Psi_{n}(z, t)= a_{0}(t) \exp \left\{\sum_{i=1}^{n} \lambda_{0 i}^{+} z_{i} t\right\} \exp \left\{\sum_{i=1}^{n} \frac{\mu_{i} p_{i 0}+\lambda_{0 i}^{-} q_{i 0}}{z_{i}} t\right\} \times \\
& \times \exp \left\{\sum_{i, j=1}^{n}\left(\mu_{i} p_{i j}^{+}+\lambda_{0 i}^{-} q_{i j}\right) \frac{z_{j}}{z_{i}} t\right\} \exp \left\{\sum_{i, j=1}^{n} \mu_{i} p_{i j}^{-} q_{j 0} \frac{1}{z_{i} z_{j}} t\right\} \times \\
& \times \exp \left\{\sum_{i, j=1}^{n} \mu_{i} \sum_{s=1}^{n} p_{i j}^{-} q_{j s} \frac{z_{s}}{z_{i} z_{j}} t\right\} \prod_{l=1}^{n} z_{l}^{\alpha_{l}}
\end{aligned}
$$

where $a_{0}(t)=\exp \left\{-\sum_{i=1}^{n}\left(\lambda_{0 i}^{+}+\lambda_{0 i}^{-}+\mu_{i}\right) t\right\}$. 
Transform (5) to a form suitable for finding the state probabilities of the network, expanding its member exhibitors in the Maclaurin series. Then the following statement

Theorem. The expression for the generating function has the form

$$
\begin{gathered}
\Psi_{n}(z, t)=a_{0}(t) \sum_{l_{1}=0}^{\infty} \ldots \sum_{l_{n}=0}^{\infty} \sum_{c_{1}=0}^{\infty} \ldots \sum_{c_{n}=0}^{\infty} \sum_{r_{1}=0}^{\infty} \ldots \sum_{r_{n}=0}^{\infty} \sum_{u_{1}=0}^{\infty} \ldots \sum_{u_{n}=0}^{\infty} \sum_{w_{1}=0}^{\infty} \ldots \sum_{w_{n}=0}^{\infty} t^{\sum_{i=1}^{n}\left(l_{i}+c_{i}+r_{i}+u_{i}+w_{i}\right)} \times \\
\times \prod_{i=1}^{n} \frac{\lambda_{0 i}^{+l_{i}}\left(\mu_{i} p_{i 0}+\lambda_{0 i}^{-} q_{i 0}\right)^{c_{i}} \prod_{j=1}^{n}\left(\mu_{i} p_{i j}^{+}+\lambda_{0 i}^{-} q_{i j}\right)^{r_{i}}\left(\mu_{i} \prod_{j=1}^{n} p_{i j}^{-} q_{j 0}\right)^{u_{i}+w_{i}}\left(\prod_{j=1}^{n} \prod_{s=1}^{n} q_{j s}\right)^{w_{i}}}{l_{i} ! c_{i} ! r_{i} ! u_{i} ! w_{i} !} \\
\times z_{i}^{\alpha_{i}+l_{i}-c_{i}-r_{i}+R-u_{i}-U-W}
\end{gathered}
$$

where $R=\sum_{i=1}^{n} r_{i}, U=\sum_{i=1}^{n} u_{i}, W=\sum_{i=1}^{n} w_{i}$.

Proof. From relation (5) follows that

$$
\Psi_{n}(z, t)=a_{0}(t) a_{1}(z, t) a_{2}(z, t) a_{3}(z, t) a_{4}(z, t) a_{5}(z, t) \prod_{l=1}^{n} z_{l}^{\alpha_{l}},
$$

where:

$$
\begin{gathered}
a_{1}(z, t)=\exp \left\{\sum_{i=1}^{n} \lambda_{0 i}^{+} z_{i} t\right\}=\prod_{i=1}^{n} \exp \left\{\lambda_{0 i}^{+} z_{i} t\right\}=\prod_{i=1}^{n} \sum_{l_{i}=0}^{\infty} \frac{\left[\lambda_{0 i}^{+} z_{i} t\right]^{l_{i}}}{l_{i} !}= \\
=\sum_{l_{1}=0}^{\infty} \ldots \sum_{l_{n}=0}^{\infty} \prod_{i=1}^{n} \frac{\left[\lambda_{0 i}^{+} z_{i} t\right]^{l_{i}}}{l_{i} !}=\sum_{l_{1}=0}^{\infty} \ldots \sum_{l_{n}=0}^{\infty} \frac{t^{l_{1}+l_{2}+\ldots+l_{n}}}{l_{1} ! l_{2} ! \ldots \cdot l_{n} !} \lambda_{01}^{l_{1}} \cdot \ldots \cdot \lambda_{0 n}^{+} l_{n} z_{1}^{l_{1}} \cdot \ldots \cdot z_{n}^{l_{n}}, \\
a_{2}(z, t)=\exp \left\{\sum_{i=1}^{n} \frac{\mu_{i} p_{i 0}+\lambda_{0 i}^{-} q_{i 0}}{z_{i}} t\right\}=\prod_{i=1}^{n} \exp \left\{\frac{\mu_{i} p_{i 0}+\lambda_{0 i}^{-} q_{i 0}}{z_{i}} t\right\}= \\
=\prod_{i=1}^{n} \sum_{c_{i}=0}^{\infty} \frac{\left[\left(\mu_{i} p_{i 0}+\lambda_{0 i}^{-} q_{i 0}\right) t z_{i}^{-1}\right]^{c_{i}}}{c_{i} !}=\sum_{c_{1}=0}^{\infty} \ldots \sum_{c_{n}=0}^{\infty} \prod_{i=1}^{n}\left[\left(\mu_{i} p_{i 0}+\lambda_{0 i}^{-} q_{i 0}\right) t z_{i}^{-1}\right]^{c_{i}} \\
=\sum_{c_{1}=0}^{\infty} \ldots \sum_{c_{n}=0}^{\infty} t^{c_{1}+\ldots+c_{n}} \frac{\left(\mu_{1} p_{10}+\lambda_{01}^{-} q_{10}\right)^{c_{1}} \cdot \ldots \cdot\left(\mu_{n} p_{n 0}+\lambda_{0 n}^{-} q_{n 0}\right)^{c_{n}}}{c_{1} ! \cdot \ldots \cdot c_{n} !} \cdot \ldots \cdot z_{n}^{-c_{n}}, \\
a_{3}(z, t)=\exp \left\{\sum_{i, j=1}^{n}\left(\mu_{i} p_{i j}^{+}+\lambda_{0 i}^{-} q_{i j}\right) \frac{z_{j}}{z_{i}} t\right\}=\prod_{i=1}^{n} \prod_{j=1}^{n} \exp \left\{\left(\mu_{i} p_{i j}^{+}+\lambda_{0 i}^{-} q_{i j}\right) \frac{z_{j}}{z_{i}} t\right\}= \\
=\prod_{i=1}^{n} \prod_{j=1}^{n} \sum_{r_{i}=0}^{\infty} \frac{\left[\left(\mu_{i} p_{i j}^{+}+\lambda_{0 i}^{-} q_{i j}\right) t z_{j} z_{i}^{-1}\right] r_{i}}{r_{i} !}=\sum_{r_{1}=0}^{\infty} \ldots \sum_{r_{n}=0}^{\infty} \prod_{i=1}^{n} \prod_{j=1}^{n}\left[\frac{\left[\left(\mu_{i} p_{i j}^{+}+\lambda_{0 i}^{-} q_{i j}\right) t z_{j} z_{i}^{-1}\right]^{r_{i}}}{r_{i} !}=\right.
\end{gathered}
$$




$$
\begin{aligned}
& =\sum_{r_{1}=0}^{\infty} \ldots \sum_{r_{n}=0}^{\infty} t^{r_{1}+\ldots+r_{n}} \frac{\left(\prod_{j=1}^{n}\left(\mu_{1} p_{1 j}^{+}+\lambda_{01}^{-} q_{1 j}\right)\right)^{n_{1}} \cdot \ldots \cdot\left(\prod_{j=1}^{n}\left(\mu_{n} p_{n j}^{+}+\lambda_{0 n}^{-} q_{n j}\right)\right)^{r_{n}}}{r_{1} ! \ldots \cdot r_{n} !} \times \\
& \times z_{1}^{r_{1}+r_{2}+\ldots+r_{n}} z_{2}^{n_{1}+r_{2}+\ldots+r_{n}} \cdot \ldots \cdot z_{n}^{r_{1}+r_{2}+\ldots+r_{n}} z_{1}^{-r_{1}} z_{2}^{-r_{2}} \ldots z_{n}^{-r_{n}}= \\
& =\sum_{n=0}^{\infty} \ldots \sum_{r_{n}=0}^{\infty} t^{r_{1}+\ldots+r_{n}} \frac{\left(\prod_{j=1}^{n}\left(\mu_{1} p_{1 j}^{+}+\lambda_{01}^{-} q_{1 j}\right)\right)^{n} \cdot \ldots \cdot\left(\prod_{j=1}^{n}\left(\mu_{n} p_{n j}^{+}+\lambda_{0 n}^{-} q_{n j}\right)\right)^{r_{n}}}{r_{1} ! \cdot \ldots \cdot r_{n} !} z_{1}^{R-r_{1}} \cdot \ldots \cdot z_{n}^{R-r_{n}}, \\
& a_{4}(z, t)=\exp \left\{\sum_{i, j=1}^{n} \mu_{i} p_{i j}^{-} q_{j 0} \frac{1}{z_{i} z_{j}} t\right\}=\prod_{i=1}^{n} \prod_{j=1}^{n} \exp \left\{\mu_{i} p_{i j}^{-} q_{j 0} \frac{1}{z_{i} z_{j}} t\right\}= \\
& =\prod_{i=1}^{n} \prod_{j=1}^{n} \sum_{u_{i}=0}^{\infty} \frac{\left[\mu_{i} p_{i j}^{-} q_{j 0} t z_{i}^{-1} z_{j}^{-1}\right]^{u_{i}}}{u_{i} !}=\sum_{u_{1}=0}^{\infty} \ldots \sum_{u_{n}=0}^{\infty} \prod_{i=1}^{n} \prod_{j=1}^{n} \frac{\left[\mu_{i} p_{i j}^{-} q_{j 0} t z_{i}^{-1} z_{j}^{-1}\right]^{u_{i}}}{u_{i} !}= \\
& =\sum_{u_{1}=0}^{\infty} \ldots \sum_{u_{n}=0}^{\infty} t^{u_{1}+\ldots+u_{n}} \frac{\left(\mu_{1} \prod_{j=1}^{n} p_{1 j}^{-} q_{j 0}\right)^{u_{1}} \cdot \ldots \cdot\left(\mu_{n} \prod_{j=1}^{n} p_{n j}^{-} q_{j 0}\right)^{u_{n}}}{u_{1} ! \ldots \cdot u_{n} !} \times \\
& \times z_{1}^{-u_{1}-u_{2}-\ldots-u_{n}} z_{2}^{-u_{1}-u_{2}-\ldots-u_{n}} \cdot \ldots \cdot z_{n}^{-u_{1}-u_{2}-\ldots-u_{n}} z_{1}^{-u_{1}} z_{2}^{-u_{2}} \ldots z_{n}^{-u_{n}}= \\
& =\sum_{u_{1}=0}^{\infty} \ldots \sum_{u_{n}=0}^{\infty} t^{u_{1}+\ldots+u_{n}} \frac{\left(\mu_{1} \prod_{j=1}^{n} p_{1 j}^{-} q_{j 0}\right)^{u_{1}} \cdot \ldots \cdot\left(\mu_{n} \prod_{j=1}^{n} p_{n j}^{-} q_{j 0}\right)^{u_{n}}}{u_{1} ! \ldots \cdot u_{n} !} z_{1}^{-\left(U+u_{1}\right)} \cdot \ldots \cdot z_{n}^{-\left(U+u_{n}\right)}, \\
& a_{5}(z, t)=\exp \left\{\sum_{i, j=1}^{n} \mu_{i} \sum_{s=1}^{n} p_{i j}^{-} q_{j s} \frac{z_{s}}{z_{i} z_{j}} t\right\}=\prod_{i=1}^{n} \prod_{j=1}^{n} \prod_{s=1}^{n} \exp \left\{\mu_{i} p_{i j}^{-} q_{j s} \frac{z_{s}}{z_{i} z_{j}} t\right\}= \\
& =\prod_{i=1}^{n} \prod_{j=1}^{n} \prod_{s=1}^{n} \sum_{u_{i}=0}^{\infty} \frac{\left[\mu_{i} p_{i j}^{-} q_{j s} t z_{i}^{-1} z_{j}^{-1} z_{s}\right]^{w_{i}}}{w_{i} !}=\sum_{w_{1}=0}^{\infty} \ldots \sum_{w_{n}=0}^{\infty} \prod_{i=1}^{n} \prod_{j=1}^{n} \prod_{s=1}^{n} \frac{\left[\mu_{i} p_{i j}^{-} q_{j s} t z_{i}^{-1} z_{j}^{-1} z_{s}\right]^{w_{i}}}{w_{i} !}= \\
& =\sum_{w_{1}=0}^{\infty} \ldots \sum_{w_{n}=0}^{\infty} t^{w_{1}+\ldots+w_{n}} \frac{\left(\mu_{1} \prod_{j=1}^{n} p_{1 j}^{-} \prod_{j=1}^{n} q_{j s}\right)^{w_{1}} \cdot \ldots \cdot\left(\mu_{n} \prod_{j=1}^{n} p_{n j}^{-} \prod_{j=1}^{n} q_{j s}\right)^{w_{n}}}{w_{1} ! \ldots \cdot w_{n} !} \times \\
& \times z_{1}^{-w_{1}-w_{2}-\ldots-w_{n}} z_{2}^{-w_{1}-w_{2}-\ldots-w_{n}} \cdot \ldots \cdot z_{n}^{-w_{1}-w_{2}-\ldots-w_{n}} z_{1}^{-w_{1}} z_{2}^{-w_{2}} \ldots z_{n}^{-w_{n}} z_{1}^{w_{1}} z_{2}^{w_{2}} \ldots z_{n}^{w_{n}}= \\
& =\sum_{w_{1}=0}^{\infty} \ldots \sum_{w_{n}=0}^{\infty} t^{w_{1}+\ldots+w_{n}} \frac{\left(\mu_{1} \prod_{j=1}^{n} p_{1 j}^{-} \prod_{j=1}^{n} q_{j s}\right)^{w_{1}} \cdot \ldots \cdot\left(\mu_{n} \prod_{j=1}^{n} p_{n j}^{-} \prod_{j=1}^{n} q_{j s}\right)^{w_{n}}}{w_{1} ! \ldots \cdot w_{n} !} z_{1}^{-W} \cdot \ldots \cdot z_{n}^{-W} .
\end{aligned}
$$


Multiplying $a_{0}(t), a_{1}(z, t), a_{2}(z, t), a_{3}(z, t), a_{4}(z, t), a_{5}(z, t)$ and $\prod_{l=1}^{n} z_{l}^{\alpha_{l}}$ we get the expression (6).

State probability of $P\left(k_{1}, k_{2}, \ldots, k_{n}, t\right)$ is the coefficient at $z_{1}^{k_{1}} z_{2}^{k_{2}}, \ldots, z_{n}^{k_{n}}$ in the expansion of function of $\Psi_{n}(z, t)$ in a multiple series of (8), on condition that at the initial time the network is in a state of $\left(\alpha_{1}, \alpha_{2}, \ldots, \alpha_{n}, 0\right)$.

\section{Finding the average number of messages at network systems}

It is known that the expectation of $m$-th components of a multi-dimensional random variable can be found by differentiating the generating function (6) by $z_{m}$ and assumed $z_{i}=1, i=\overline{1, n}$. Therefore, the average number of messages in the system $S_{m}$ of network we will use the relation:

$$
\begin{gathered}
\frac{\partial \Psi_{n}(z, t)}{\partial z_{m}}=\sum_{l_{1}=0}^{\infty} \ldots \sum_{l_{n}=0}^{\infty} \sum_{c_{1}=0}^{\infty} \ldots \sum_{c_{n}=0}^{\infty} \sum_{n=0}^{\infty} \ldots \sum_{r_{n}=0}^{\infty} \\
\sum_{u_{1}=0}^{\infty} \ldots \sum_{u_{n}=0}^{\infty} \sum_{w_{1}=0}^{\infty} \ldots \sum_{w_{n}=0}^{\infty}\left(\alpha_{m}+l_{m}-c_{m}-r_{m}+R-u_{m}-U-W\right) t^{\sum_{i=1}^{n}\left(l_{i}+c_{i}+r_{i}+u_{i}+w_{i}\right)} \times \\
\times \prod_{i=1}^{n} \frac{\lambda_{0 i}^{+} l_{i}\left(\mu_{i} p_{i 0}+\lambda_{0 i}^{-} q_{i 0}\right)^{c_{i}} \prod_{j=1}^{n}\left(\mu_{i} p_{i j}^{+}+\lambda_{0 i}^{-} q_{i j}\right)^{r_{i}}\left(\mu_{i} \prod_{j=1}^{n} p_{i j}^{-} q_{j 0}\right)^{u_{i}+w_{i}}\left(\prod_{j=1}^{n} \prod_{s=1}^{n} q_{j s}\right)^{w_{i}}}{l_{i} ! c_{i} ! r_{i} ! u_{i} ! w_{i} !} \times \\
\times z_{m}^{\alpha_{m}+l_{m}-c_{m}-r_{m}+R-u_{m}-U-W-1} \prod_{\substack{i=1, i \neq m}}^{n} z_{i}^{\alpha_{i}+l_{i}-q_{i}-r_{i}+R-u_{i}-U-W} .
\end{gathered}
$$

It follows that the average number of messages in the system $S_{m}$, is defined by the formula

$$
\begin{gathered}
N_{m}(t)=\left.\frac{\partial \Psi_{n}(z, t)}{\partial z_{m}}\right|_{z=(1,1, \ldots, 1)}=a_{0}(t) \sum_{l_{1}=0}^{\infty} \ldots \sum_{l_{n}=0}^{\infty} \sum_{c_{1}=0}^{\infty} \ldots \sum_{c_{n}=0}^{\infty} \sum_{r_{1}=0}^{\infty} \ldots \sum_{r_{n}=0}^{\infty} \\
\sum_{u_{1}=0}^{\infty} \ldots \sum_{u_{n}=0}^{\infty} \sum_{w_{1}=0}^{\infty} \ldots \sum_{w_{n}=0}^{\infty}\left(\alpha_{m}+l_{m}-c_{m}-r_{m}+R-u_{m}-U-W\right) \sum^{\sum_{i=1}^{n}\left(l_{i}+c_{i}+r_{i}+u_{i}+w_{i}\right)} \times \\
\times \prod_{i=1}^{n} \frac{\lambda_{0 i}^{+} l_{i}\left(\mu_{i} p_{i 0}+\lambda_{0 i}^{-} q_{i 0}\right)^{c_{i}} \prod_{j=1}^{n}\left(\mu_{i} p_{i j}^{+}+\lambda_{0 i}^{-} q_{i j}\right)^{r_{i}}\left(\mu_{i} \prod_{j=1}^{n} p_{i j}^{-} q_{j 0}\right)^{u_{i}+w_{i}}\left(\prod_{j=1}^{n} \prod_{s=1}^{n} q_{j s}\right)^{w_{i}}}{l_{i} ! c_{i} ! r_{i} ! u_{i} ! w_{i} !} .
\end{gathered}
$$


We make the expression (7) the change of variables

$$
k_{m}=\alpha_{m}+l_{m}-c_{m}-r_{m}+R-u_{m}-U-W,
$$

then $l_{m}=k_{m}-\alpha_{m}+c_{m}+r_{m}-R+u_{m}+U+W$ and

$$
\begin{aligned}
& N_{m}(t)=a_{0}(t) \sum_{c_{1}=0}^{\infty} \ldots \sum_{c_{n}=0}^{\infty} \sum_{r_{1}=0}^{\infty} \ldots \sum_{r_{n}=0}^{\infty} \\
& \sum_{u_{1}=0}^{\infty} \ldots \sum_{u_{n}=0}^{\infty} \sum_{w_{1}=0}^{\infty} \ldots \sum_{w_{n}=0}^{\infty} \sum_{k_{1}=\alpha_{1}-c_{1}-r_{1}+R-u_{1}-U-W}^{\infty} \ldots \sum_{k_{n}=\alpha_{n}-c_{n}-r_{n}+R-u_{n}-U-W}^{\infty} k_{m} \times \\
& \times t^{i=1} k_{i}^{n}\left(\alpha_{i}+2 c_{i}+2 r_{i}-R+2 u_{i}+U+W\right) \times \\
& \times \prod_{i=1}^{n} \frac{\lambda_{0 i}^{+l_{i}}\left(\mu_{i} p_{i 0}+\lambda_{0 i}^{-} q_{i 0}\right)^{c_{i}} \prod_{j=1}^{n}\left(\mu_{i} p_{i j}^{+}+\lambda_{0 i}^{-} q_{i j}\right)^{r_{i}}\left(\mu_{i} \prod_{j=1}^{n} p_{i j}^{-} q_{j 0}\right)^{u_{i}+w_{i}}\left(\prod_{j=1}^{n} \prod_{s=1}^{n} q_{j s}\right)^{w_{i}}}{\left(k_{i}-\alpha_{i}+c_{i}+r_{i}-R+u_{i}+U+W\right) ! q_{i} ! r_{i} ! u_{i} ! w_{i} !} .
\end{aligned}
$$

Because network systems operate under high load, then

$$
k_{i}=\alpha_{i}-c_{i}-r_{i}+R-u_{i}-U-W \geq 1
$$

and, therefore, $c_{i} \leq \alpha_{i}-r_{i}+R-u_{i}-U-W-1$, so

$$
\begin{aligned}
& N_{m}(t)=a_{0}(t) \sum_{k_{1}=0}^{\infty} \ldots \sum_{k_{m}=0}^{\infty} k_{m} \ldots \sum_{k_{n}=0}^{\infty} \sum_{r_{1}=0}^{\infty} \ldots \sum_{r_{n}=0}^{\infty} \\
& \sum_{u_{1}=0}^{\infty} \ldots \sum_{u_{n}=0}^{\infty} \sum_{w_{1}=0}^{\infty} \ldots \sum_{w_{n}=0}^{\infty} \sum_{c_{1}=0}^{\alpha_{1}-r_{1}+R-u_{1}-U-W-1} \cdots \sum_{c_{n}=0}^{\alpha_{n}-r_{n}+R-u_{n}-U-W-1} t^{\sum_{i=1}^{n}\left(k_{i}-\alpha_{i}+2 c_{i}+2 r_{i}-R+2 u_{i}+U+W\right)} \\
& \times \prod_{i=1}^{n} \frac{\lambda_{0 i}^{+} l_{i}\left(\mu_{i} p_{i 0}+\lambda_{0 i}^{-} q_{i 0}\right)^{c_{i}} \prod_{j=1}^{n}\left(\mu_{i} p_{i j}^{+}+\lambda_{0 i}^{-} q_{i j}\right)^{r_{i}}\left(\mu_{i} \prod_{j=1}^{n} p_{i j}^{-} q_{j 0}\right)^{u_{i}+w_{i}}\left(\prod_{j=1}^{n} \prod_{s=1}^{n} q_{j s}\right)^{w_{i}}}{\left(k_{i}-\alpha_{i}+c_{i}+r_{i}-R+u_{i}+U+W\right) ! c_{i} ! r_{i} ! u_{i} ! w_{i} !} .
\end{aligned}
$$

To find state probabilities and the average number of messages in a network systems a program was written in a mathematical computation package Mathematica.

\section{Examples}

Let the number of QS in the network $n=3$. The intensity of the input stream of positive and signals $\lambda_{0 i}^{+}$and $\lambda_{0 i}^{-}$are equal respectively to: $\lambda_{01}^{+}=1 / 2, \lambda_{02}^{+}=1 / 5$, 
$\lambda_{03}^{+}=1 / 3, \lambda_{01}^{-}=1 / 3, \lambda_{02}^{-}=1 / 4, \lambda_{03}^{-}=1 / 6$. The intensities of message service $\mu_{i}$ equal: $\mu_{1}=1 / 2, \mu_{2}=4, \mu_{3}=1 / 3$. We assume, that the transition probability of positive messages $p_{i j}^{+}$have form: $p_{12}^{+}=1 / 9, p_{13}^{+}=2 / 7, p_{21}^{+}=1 / 8, p_{23}^{+}=1 / 5$, $p_{31}^{+}=2 / 9, p_{32}^{+}=1 / 7$; the transition probability of signals $p_{i j}^{-}$equals: $p_{12}^{-}=1 / 8$, $p_{13}^{-}=1 / 7, p_{21}^{-}=3 / 7, p_{23}^{-}=1 / 9, p_{31}^{-}=2 / 7, p_{32}^{-}=2 / 11$; output probability from network of messages to the external environment $p_{i 0}$ equals $p_{10}=169 / 504$, $p_{20}=341 / 2520, p_{30}=116 / 693$. Suppose also, that the probability that the incoming signal immediately moves the positive message from the system $S_{j}$ to system $S_{s}$ equal $q_{12}=1 / 3, q_{13}=1 / 3, q_{21}=1 / 4, q_{23}=1 / 5, q_{31}=1 / 3, q_{32}=1 / 7$. The signal is triggered by a negative message and is destroyed at the QS $S_{j}$ positive message with probability $q_{10}=1 / 3, q_{20}=11 / 20, q_{30}=11 / 21$. Expression (7) in this case is equal to $a_{0}(t)=e^{-\frac{397 t}{60}}$.

For example, we need to find the probability of the state $P(3,3,3, t)$. It is the coefficient of $z_{1}{ }^{3} z_{2}{ }^{3} z_{3}{ }^{3}$ in the expansion of $\Psi_{n}(z, t)$ in multiple series (6), so the degrees at $z_{i}$ must satisfy the relation $\alpha_{i}+l_{i}-c_{i}-r_{i}+R-u_{i}-U-W=3, i=\overline{1,3}$, this implies that

$$
\begin{gathered}
c_{i}=\alpha_{i}+l_{i}+\sum_{\substack{j=1 \\
j \neq i}}^{3} r_{j}-\sum_{\substack{j=1 \\
j \neq i}}^{3} u_{j}-\sum_{j=1}^{3} w_{j}-3, \\
\sum_{i=1}^{3}\left(l_{i}+c_{i}+r_{i}+u_{i}+w_{i}\right)=\sum_{i=1}^{3}\left(\alpha_{i}+2 l_{i}\right)+3(R-U-W-3), i=\overline{1,3} .
\end{gathered}
$$

Then from (6) we obtain that

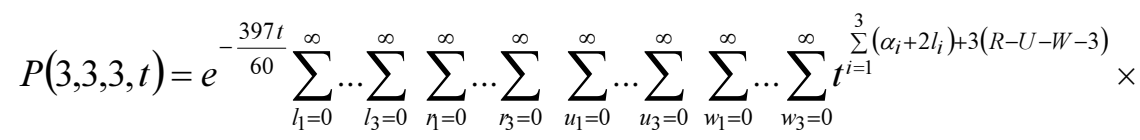

$$
\begin{aligned}
& \times \prod_{i=1}^{3} \frac{\lambda_{0 i}^{+l_{i}}\left(\mu_{i} p_{i 0}+\lambda_{0 i}^{-} q_{i 0}\right)^{\alpha_{i}+l_{i}+R-U-u_{i}-W-10}}{l_{i} !\left(\alpha_{i}+l_{i}+R-U-u_{i}-W-3\right) ! r_{i} ! u_{i} ! w_{i} !} \times \\
& \times \prod_{j=1}^{3}\left(\mu_{i} p_{i j}^{+}+\lambda_{0 i}^{-} q_{i j}\right)^{r_{i}}\left(\mu_{i} \prod_{j=1}^{3} p_{i j}^{-} q_{j 0}\right)^{u_{i}+w_{i}}\left(\prod_{j=1}^{3} \prod_{s=1}^{3} q_{j s}\right)^{w_{i}}, \alpha_{i}=3, i=\overline{1,3} .
\end{aligned}
$$

Figure 1 shows plots of the state probability of $P(3,3,3, t)$ and in condition that, at the initial time moment network is in one of three states: 1) $\alpha_{i}=3, i=\overline{1,3}$, 2) $\left.\alpha_{i}=1, i=\overline{1,3}, 3\right) \alpha_{i}=0, i=\overline{1,3}$. 


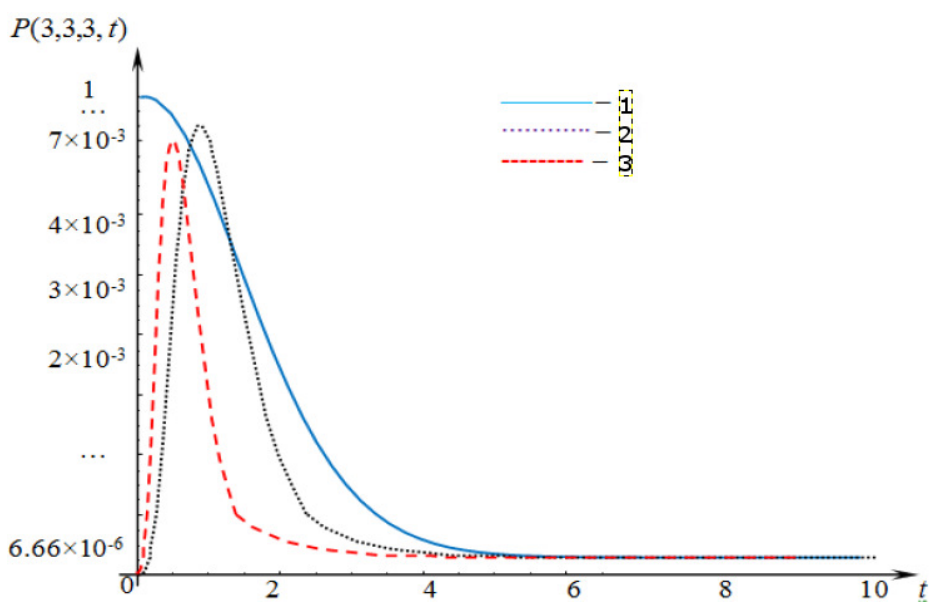

Fig. 1. Graph of state probability $P(3,3,3, t)$

We find the state probabilities and the average number of messages in network systems at the stationary behavior. For G-networks with negative messages and triggers the stationary distribution $P(k)$ appears in the multiplicative form $[3,7]$ :

$$
P(k)=\prod_{i=1}^{n} p\left(k_{i}\right), p\left(k_{i}\right)=\left(1-q_{i}\right) q_{i}^{k_{i}}, q_{i}<1, k_{i} \geq 0,
$$

where

$$
\begin{gathered}
q_{i}=\frac{\lambda_{i}^{+}}{\lambda_{i}^{-}\left(1-q_{i 0}\right)+\lambda_{i}^{-} q_{i 0}+\mu_{i}}, \\
\lambda_{i}^{+}=\lambda_{0 i}^{+}+\sum_{j=1}^{n} q_{j} \mu_{j}\left[p_{j i}^{+}+\sum_{s=1}^{n} p_{j s}^{-} q_{s} q_{s i}\right]+\sum_{j=1}^{n} \lambda_{0 j}^{-} q_{j} q_{j i}, \\
\lambda_{i}^{-}=\lambda_{0 i}^{-}+\sum_{j=1}^{n} q_{j} \mu_{j} p_{j i}^{-}, i=\overline{1, n} .
\end{gathered}
$$

The average number of messages located in system $S_{m}$, is calculated as follows:

$$
N_{m}=\sum_{k_{1}=0 k_{2}=0}^{\infty} \ldots \sum_{k_{m}=0}^{\infty} k_{m} \ldots \sum_{k_{n}=0}^{\infty} P(k), m=\overline{1, n} .
$$

The calculations in the package Mathematica showed that the stationary probabilities of the above conditions are equal to respectively $P(3,3,3)=6.66 \times 10^{-6}$, $P(1,1,1)=0,003, P(0,0,0)=0,07$, and the average number messages is equal to respectively $N_{1}=0.0004, N_{3}=0.0003, N_{3}=0.0006$. 
The average number of requests in the system network (in the queue and on service) on condition that $N_{m}(0)=0, m=\overline{1, n}$, can be found by the formula (8).

Figure 2 shows a graph of the average number of messages in the QS $S_{1}$ in the network form example above.

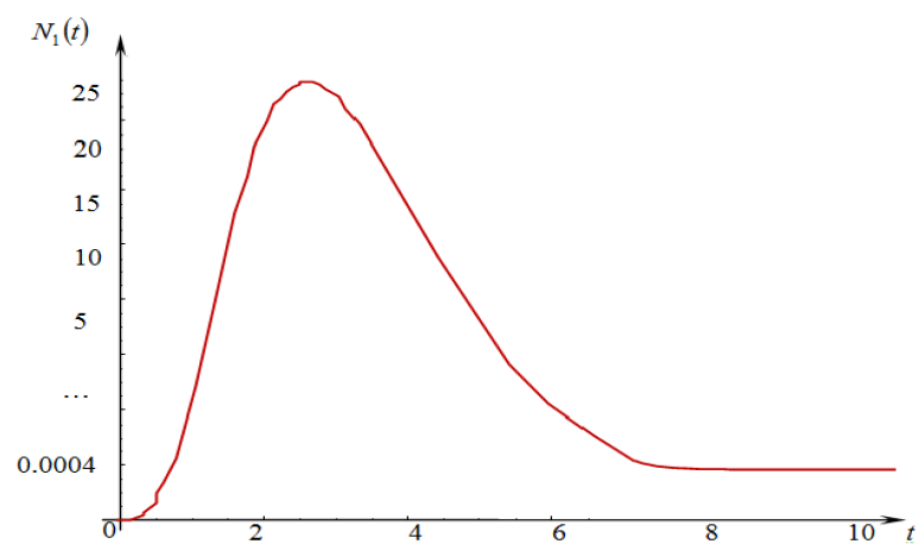

Fig. 2. Graph change in the average number of messages $N_{1}(t)$ in QS $S_{1}$

\section{References}

[1] Naumenko V., Matalytski M., Network analysis with positive and negative messages at transient behavior [Analiz seti s polozhitel'nymi i otritsatel'nymi zayavkami v perekhodnom rezhime], Vestnik of Yanka Kupala State University of Grodno. Series 2. Mathematics. Physics. Informatics, Computer Technology and its Control 2013, 3 (159), 135-142 (in Russian).

[2] Matalytski M., Naumenko V., Nonstationary analysis of queueing network with positive and negative messages, Journal of Applied Mathematics and Computational Mechanics 2013, 2, 12, 61-71.

[3] Gelenbe E., G-networks with triggered customer movement, Journal of Applied Probability 1993, 30, $742-748$.

[4] Gelenbe E., G-networks with signals and batch removal, Prob. Eng. Inf. Sci. 1993, 7, 335-342.

[5] Henderson W., Northcote B.S., Taylor P.G., State-dependent signalling in queueing networks, Advanced of Applied Probability 1994, 26, 436-455.

[6] Gelenbe E., Pujolle G., Introduction to Queueing Networks, John Wiley, New York 1998, 244 p.

[7] Bocharov P., Vishnevsky V., G-networks: the development of the theory of multiplicative networks [G-seti: razvitiye teorii mul'tiplikativnykh setey], Automation and Remote Control 2003, 5, 46-74 (in Russian). 